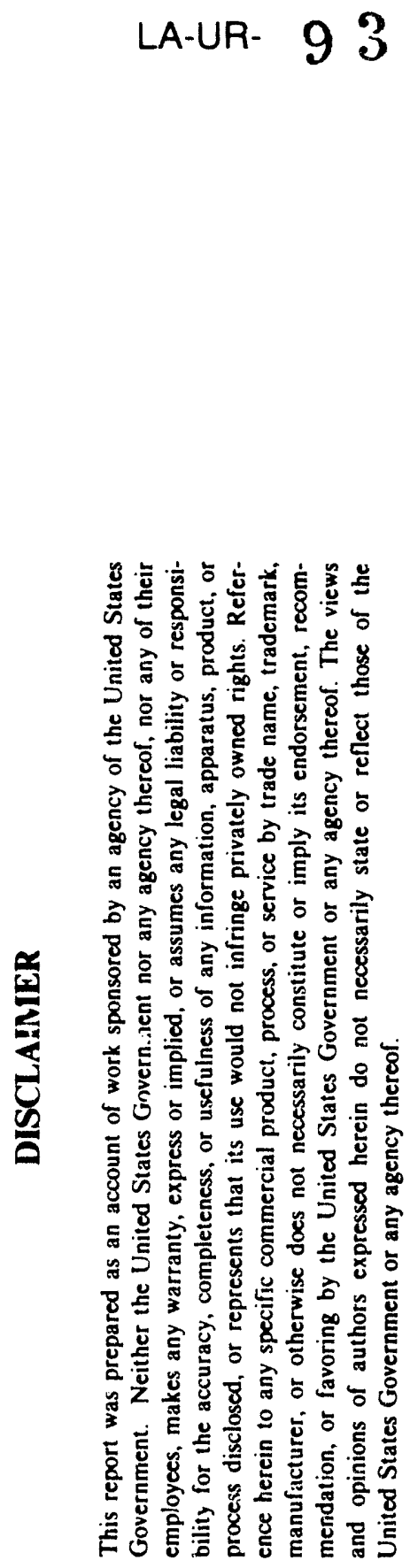

\section{5 \\ Title:}

Author(s):

Submitted to:

MICROTEXTURAL CHARACTERIZATION OF ANNEALED

AND DEFORMED COPPER

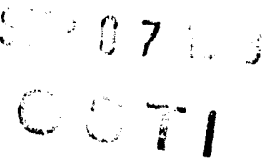

S. I. Wright, MST-6

F. Heidelbach, CMS (GRA at UC Berkeley)

International Conference on Textures of Materials Clausthal, Germany

September 20-24, 1993

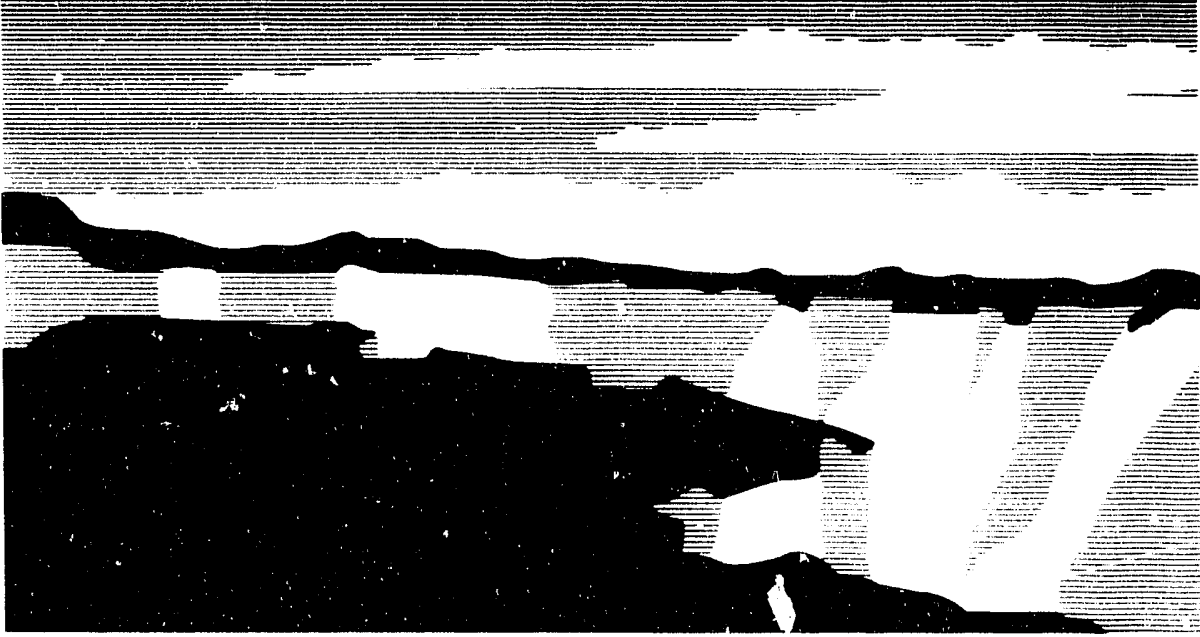

Los Alamos National Laboratory, an aftirmative actior.'equal opportunity employer, is operated by the University of California for the U.S. Department of Energy under contract W-7405-ENG-36. By acceptance of this article, the publisher recognizes hat the U.S. Government retains a nonexclusive, royalty-free license to publish or reproduce the published form of this contribution, or to allow othors to do so, for U.S. Government purposes. The Los Alamos ilational Laboratory requests that the publisher identify this article as work pertormed under the auspices of the U.S. Department of Energy. 


\title{
MICROTEXTURAL CHARACTERIZATION OF ANNEALED AND DEFORMED COPPER
}

\author{
S. I. Wright and F. Heidelbach \\ Los Alamos National Laboratory, Los Alamos, NM 87545
}

\section{Introduction}

Grain boundary textures of copper in several different states are examined using backscatter electron Kikuchi diffraction patterns (BEKPs) in the scanning electron microscope (SEM) (see review by Dingley and Randle (1)). Local orientation relationships across a former recrystallization twin boundary after deformation are explored in detail using automatic BEKP analysis (see recent works by Adams et al. (2) and Wright (3)).

\section{Experimental Details}

The starting material was high purity copper (99.99\%) which was annealed at $600^{\circ} \mathrm{C}$ for $30 \mathrm{~min}$. Samples of the starting material were rolled to $50 \%$ and $90 \%$ height reductions. The $90 \%$ rolled sample was subsequently recrystallized at $175^{\circ} \mathrm{C}$ for 1 hour. Samples were prepared from the starting material, the 50\% rolled material and the recrystallized material. We shall term these samples annealed, deformed and recrystallized to facilitate discussion.

$\mathrm{X}$-ray pole figures were obtained from each sample and used to determine orientation distributions using the WIMV (4) algorithm as implemented in popLA (5), a texture analysis software package. The annealed and recrystallized samples exhibited random textures. The deformed sample exhibited a $\langle 110\rangle$ fiber texture with a maximum at 5.14 times random. The general rolling texture components were also present in the texture; however a classic rolling texture was not produced because the rolled sample was too small for general rolling conditions to exist. There was significant strain in the transverse direction.

Grain maps covering about $1 \mathrm{~mm}^{2}$ were constructed for each of the three samples. Using BEKPs, the orientations of 333 grains were measured in the annealed sample, 200 grains in the deformed sample and 305 grains in the recrystallized sample. The grain maps were coupled with the measured orientations to determine the lattice misorientations across 881 boundaries in the annealed sample, 504 boundaries in the deformed sample and 706 boundaries in the recrystallized sample.

Approximately ten thousand automatic BEKP measurements were made in the neighborhood of what appears to be a former annealing twin in the deformed sample. The orientation measurements were made on a regular hexagonal grid with 1 micron spacing between measurements. At each point on the grid the orientation (in Euler angles according to Bunge's (6) formalism) and position of the electron beam on the sample were recorded.

\section{Misorientation Distributions}

The distribution of minimum misorientation angle for grain boundaries measured in the annealed and deformed samples is compared with a random misorientation distribution $(7,8)$ in figure 1 . The "random" distribution is based on the crystallographic texture. This was generated by forming all possible orientation pairs from the measurements in each set and then calculating the misorientation angle for each pair. The two maxima in the annealed sample correspond to primary and secondary annealing twins. The axis-angle pairs describing these twins are a $60^{\circ}$ rotation about the $\langle 111\rangle$ axis (the primary annealing twin) and a $40^{\circ}$ rotation about the $\langle 110\rangle$ axis. The secondary twin is produced when two different primary twins of the same grain share a common boundary. 
Annealed

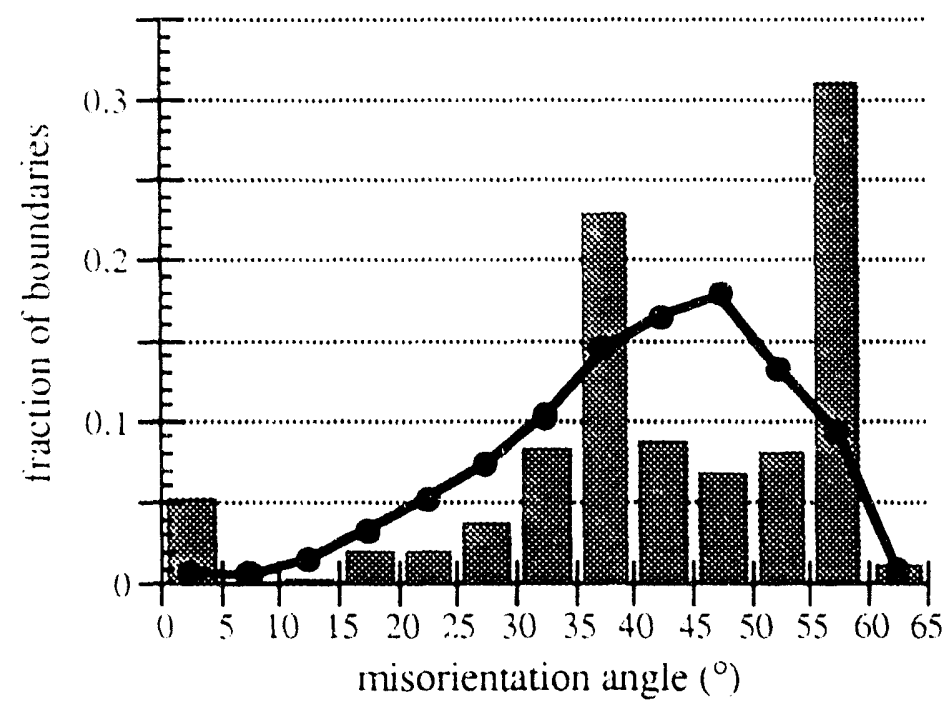

Deformed

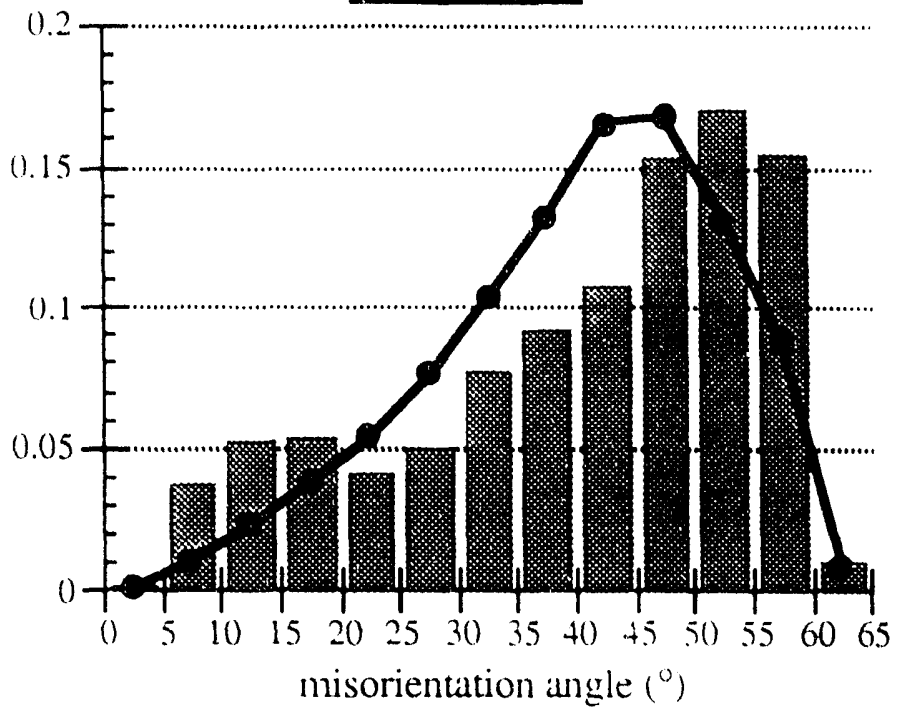

Figure 1 - Distribution of minimum misorientation angle in annealed and deformed copper.

Misorientation distribution functions (MDF) (see (9). for example) were calculated for each sample from the single orientation data. The generalized spherical harmonic series expansion method of Bunge (6) was followed. The calculation of the MDF was carried out to an order of $l=20$. Each misorientation was represented by a Gaussian with a $5^{\circ}$ half-width (10). The results of the MDF calculations are shown in figure 2.

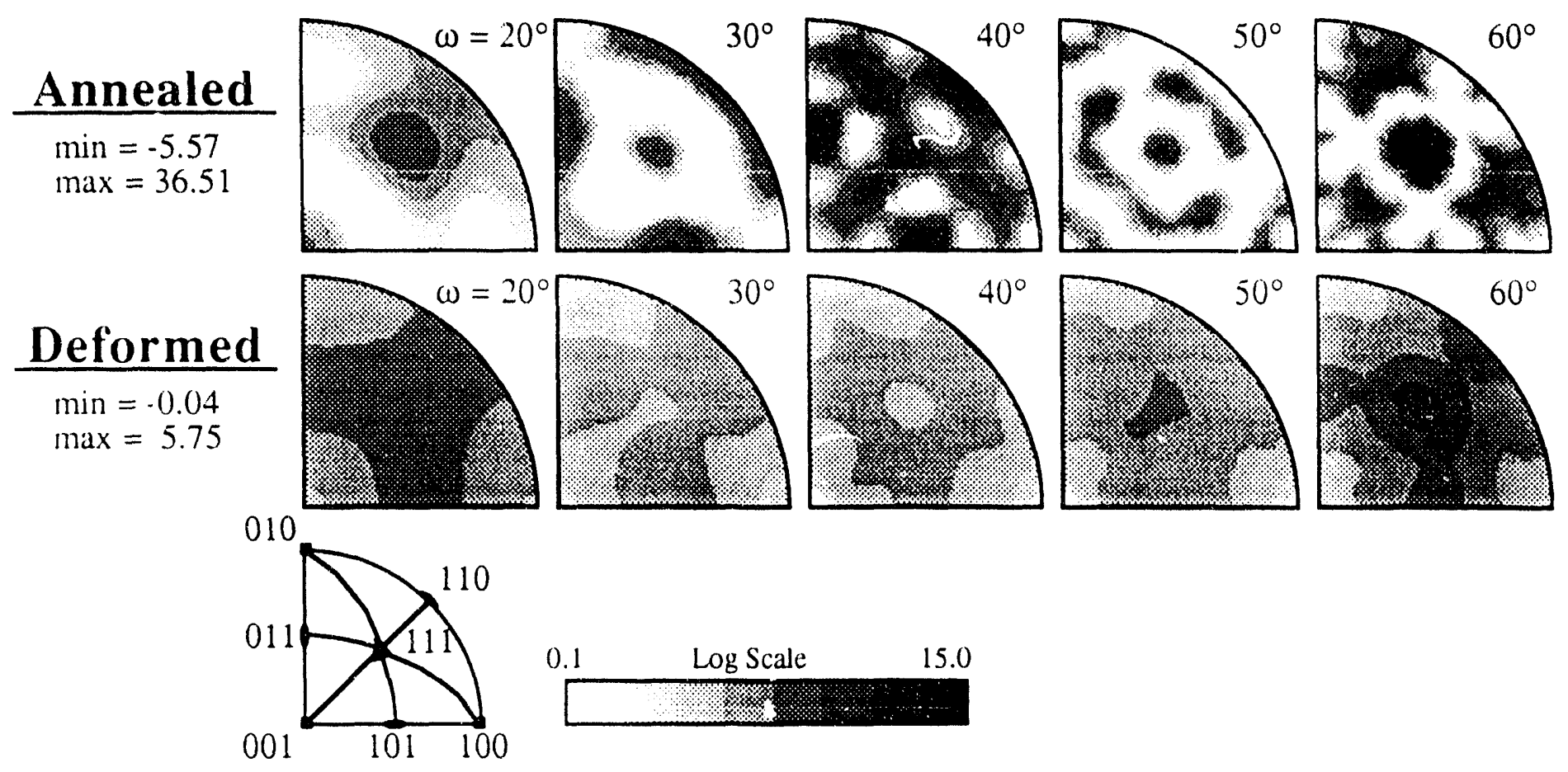

Figure 2 - Misorientation distribution function of annealed and deformed copper plotted in axis-angle space.

It appears in figures 1 and 2 that the imposed deformation breaks down the twin structures into boundaries of random misorientation. There appears to be a remnant of the original annealing twins in the deformed sample. However, the misorientation texture is considerably weaker in the deformed sample. It is interesting to note that a strong texture does not necessarily imply a strong misorientation texture. In this example the opposite is true. The deformed sample had the strongest texture of the three samples; whereas, this sample exhibited the weakest misorientation texture. 
For the sake of brevity, the results of the misorientation calculations obtained from the recrystallized sample have been omitted. Despite significant differences in the grain size distributions, the misorientation distributions of the annealed and recrystallized samples are nearly identical.

\section{Twin Boundary Characterization}

Figure 3 shows a reconstruction of the grain boundaries from the automatic BEKP measurements on the deformed material. Thick lines are drawn between neighboring measurement points with misorientation angles exceeding $15^{\circ}$ and thin lines are drawn between neighboring measurements with misorientation angles less than or eyual to $15^{\circ}$ and greater than $6^{\circ}$.

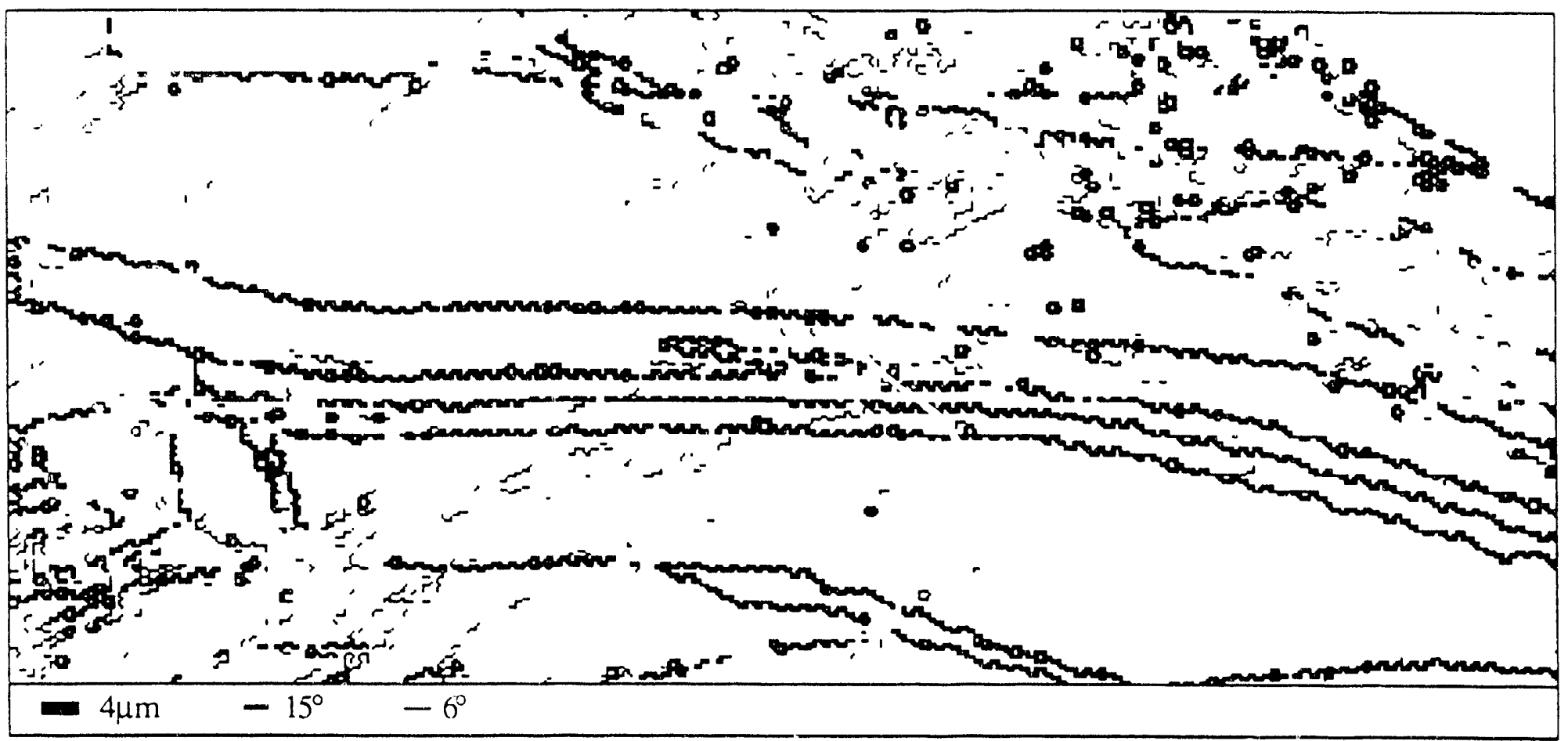

Figure 3 - Grain boundary map reconstructed from automatic BEKP measurements on deformed copper.

Figure 4 shows a schematic of the measured region. The grains labeled $\mathbf{A}$ and $\mathbf{B}$ appear to be former recrystallization twins.

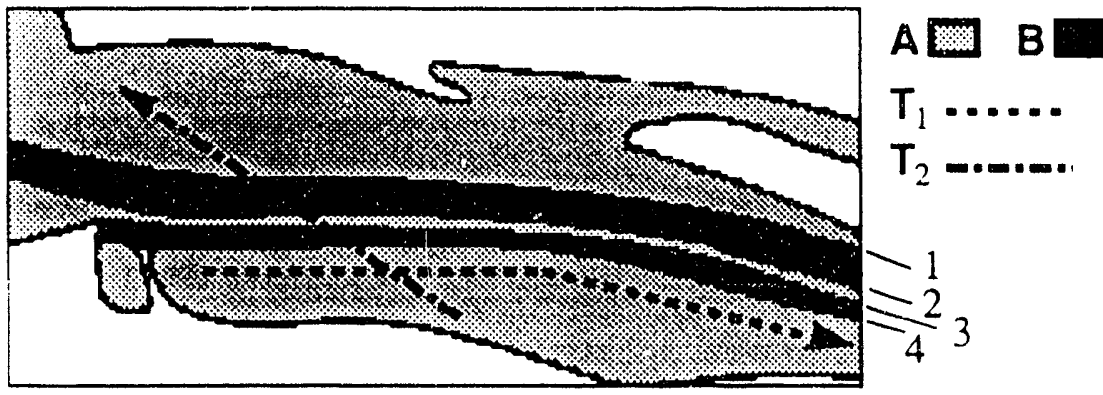

Figure 4 - Schematic of figure 3 showing twins, twin boundaries and trace lines.

An intergranular trace (denoted by $T_{1}$ in figure 4) and a transgranular trace (denoted by $T_{2}$ ) were formed by collecting all measured orientations within $1.5 \mu \mathrm{m}$ of prescribed lines though the measurement space. The misorientation angles between neighboring points on the two traces as well as the misorientation angles between the first point on the two traces and each successive point are displayed in figure 5. It should be noted that there is a fairly large spread in orientation (nearly $20^{\circ}$ ) within the grains as can be observed in the first trace. 

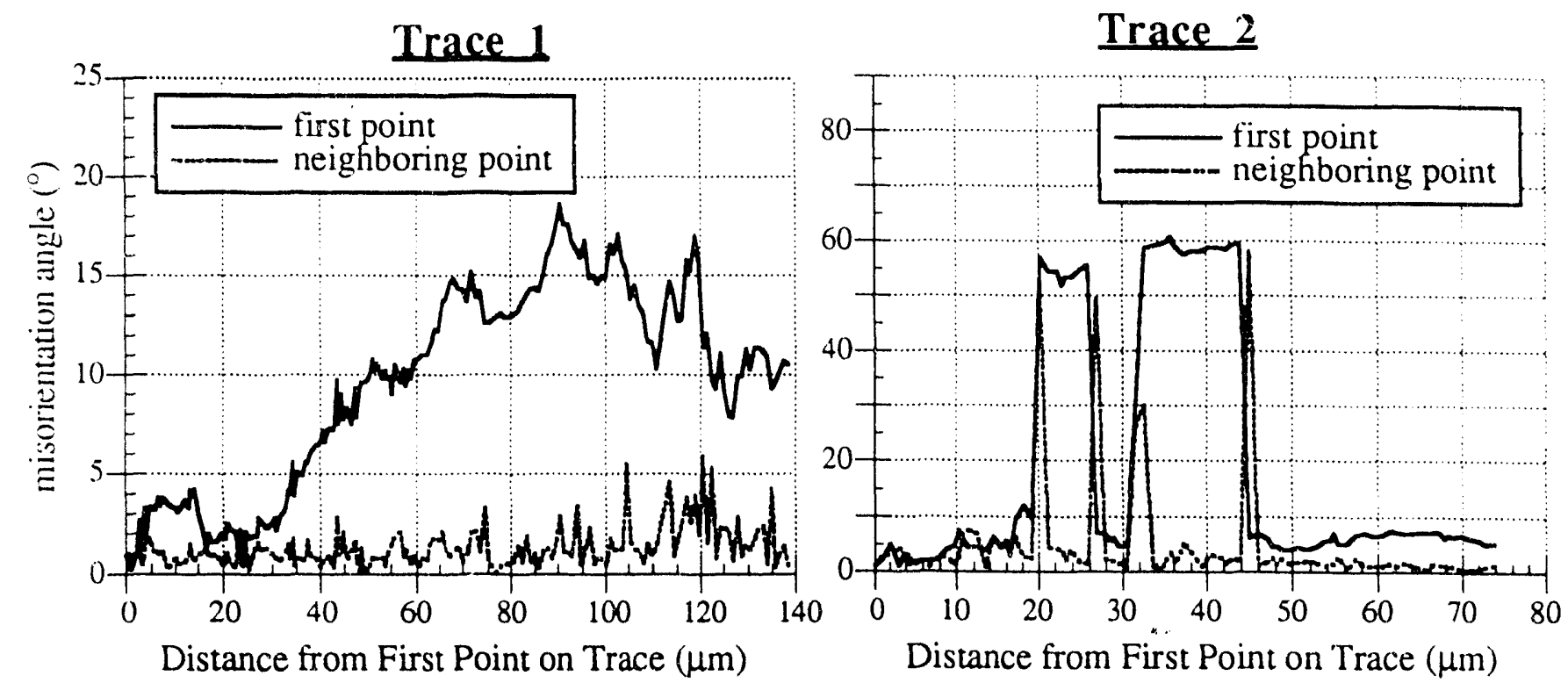

Figure 5 - Misorientations between neighboring measurements and between each measurement and the starting measurement along trace lines in deformed copper

In order to examine more closely the spatial distribution of orientation within the individual grains, the average orientations for both the $\mathbf{A}$ and the $\mathbf{B}$ grains were determined by calculating an orientation distribution function (ODF) from the automatic orientation measurements using the harmionic analysis described previously $(l=16)$. The locations in Euler space of the two highest peaks were found and used as reference orientations. The deviations of the measured orientations from these reference orientations were mapped onto a gray scale as shown in figure 6 . In this image dark areas correspond to areas very near the reference orientations; whereas, white areas have orientations greater than $15^{\circ}$ from the reference orientations.

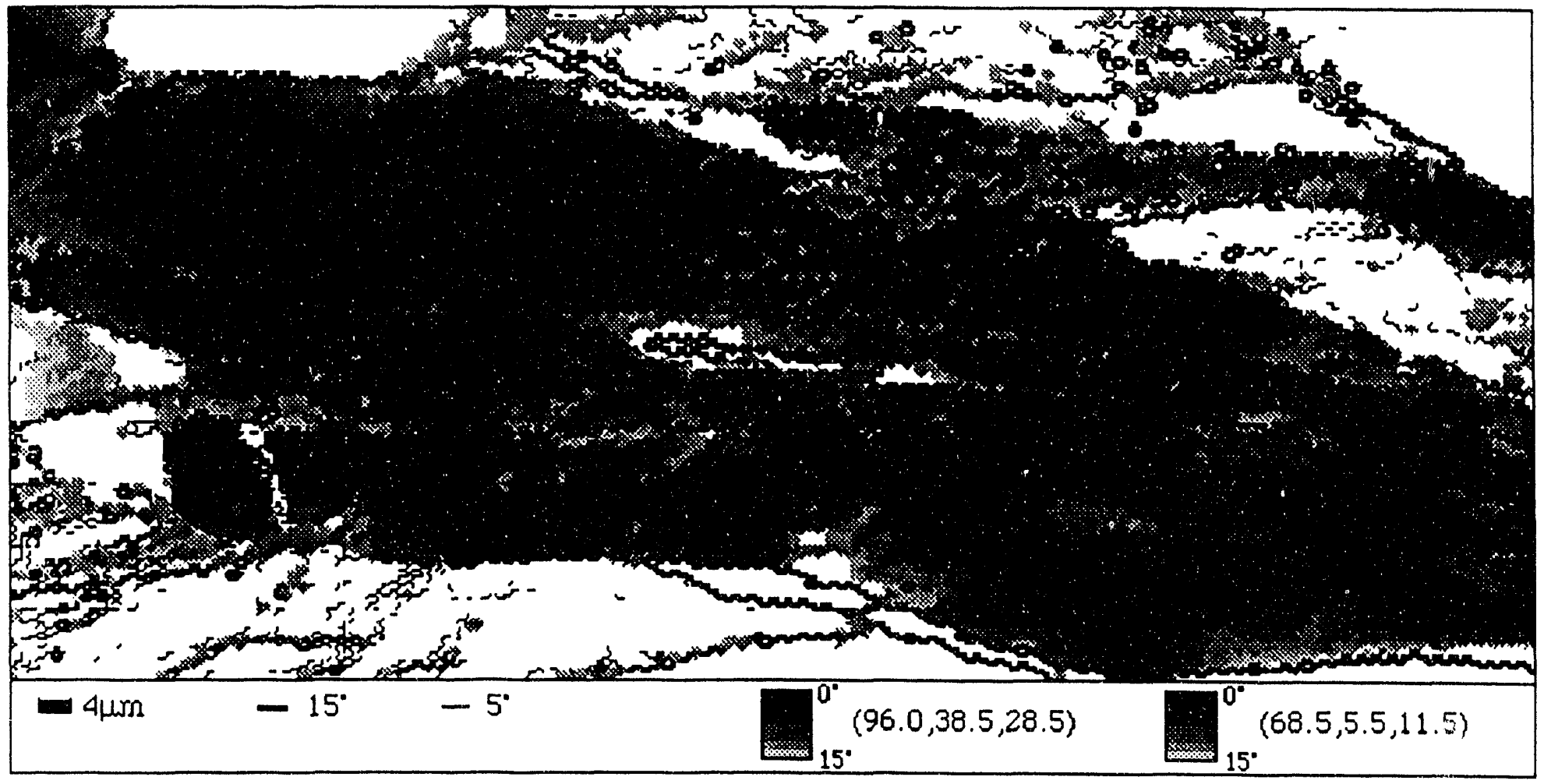

Figure 6 - Gray scale image formed by deviation of measured orientations from reference orientations

Misorientations along the boundaries labeled 1,2,3 and 4 in figure 4 were examined in detail. Misorientations greater than $5^{\circ}$ across boundary 1 are plotted in axils-angle space in figure 7 . The results for the other three boundaries are very similar. The boundary is clearly no longer a classic $<111\rangle$ twin. It appears to have broken up into a $\langle 110\rangle$ 
com

' Component and an approximate $<112>$ component. Only one "twin" bounaary is examined here; the general break

- down of all twin boundaries into specific components is unlikely since the MDF calculated for the deformed sample (see figure 2) shows no evidence of preferred misorientation around the components occurring in the case detailed here.

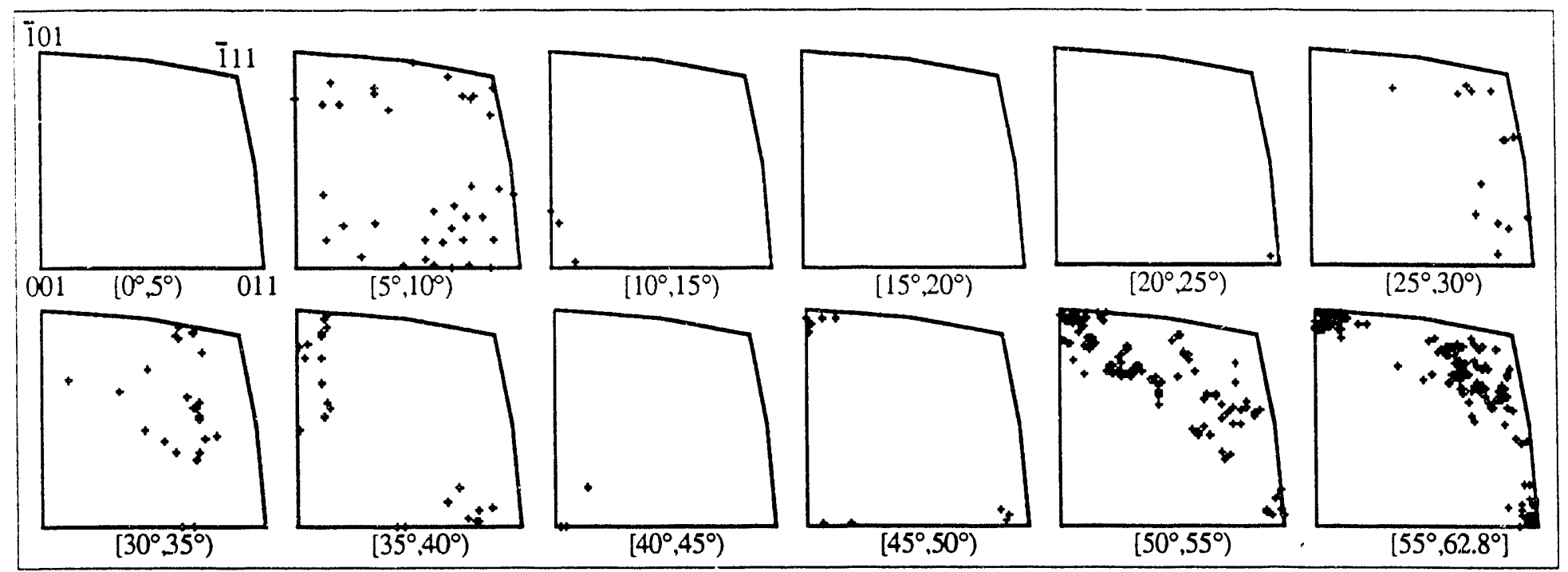

Figure 7 - Misorientations along grain boundary 1 in the deformed copper sample

The spatial distribution of misorientation along the grain boundary was examined by partitioning the boundary into four segments containing an equal number of measured misorientations. No evidence for a systematic spatial distribution of misorientations along the boundary was observed.

\section{Discussion and Conclusions}

Even though deformation produces material with preferred orientation it appears to randomize the grain boundary texture in copper. It appears that as dislocations move through the material the resulting lattice rotations serve to break down the twin structure in a stochastic manner. An effort was made to use the automatic BEKP data to search for dislocation cell wall structures. However, no evidence was found for such structures. This may be due, in part, to the resolution of the BEKP technique. Diffraction occurs from an area with mean diameter of approximately $0.5 \mu \mathrm{m}$.

\section{Acknowledgments}

The authors acknowledge K. Kunze and B. L. Adams for allowing the authors access to the automatic BEKP analysis system at Brigham Young University. The help of U. F. Kocks, R. Wenk and S. R. Chen is also gratefully acknowledged.

\section{References}

1. D. J. Dingley and V. Randle, J. Mat. Sci. 27, 4545 (1992).

2. B. L. Adams, S. I. Wright and K. Kunze, Met. Trans., (in press) (1993).

3. S. I. Wright, Journal of Computer Assisted Microscopy, (in press) (1993).

4. S. Matthies and G. W. Vinel, phys. stat. sol. (b) 112, K111 (1982).

5. J. S. Kallend, U. F. Kocks, A. D. Rollett and H.-R. Wenk, Mat. Sci. Eng. A132, 1 (1991).

6. H.-J. Bunge, Texture Analysis in Materials Science. Mathematical Methods., Butterworths, London (1982).

7. J. K. Mackenzie and M. J. Thomson, Biometrika 44, 205 (1957).

8. D. C. Handscomb, Canadian Journal of Mathematics 10, 85 (1958).

9. J. Pospiech, K. Sztwiertnia and F. Haessner, Textures and Microstructures 6, 201 (1986).

10. F. Wagner, H. R. Wenk, C. Esling and H. J. Bunge, phys. stat. sol. (b) 67, 269 (1981). 

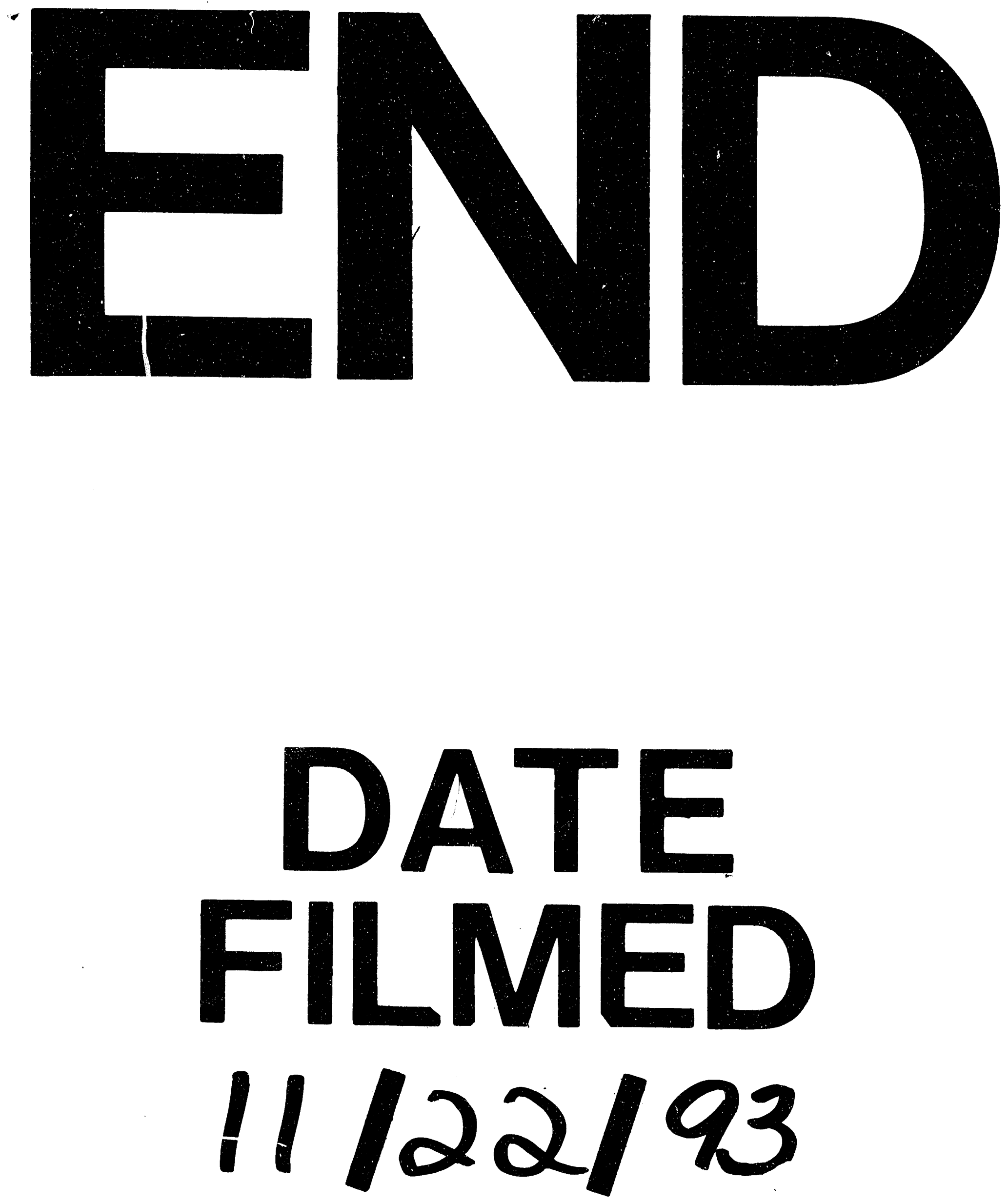
